\title{
Watermark Detection in Impulsive Noise Environment Based on the Compressive Sensing Reconstruction
}

\author{
Budimir LUTOVAC, Milos DAKOVIC, Srdjan STANKOVIC, Irena OROVIC \\ University of Montenegro, Faculty of Electrical Engineering, Dz. Vasingtona bb, 81000 Podgorica , Montenegro \\ \{budo, milos, srdjan, irenao\}@ac.me
}

Submitted October 31, 2016 / Accepted February 27, 2017

\begin{abstract}
The watermark detection procedure for images corrupted by impulsive noise is proposed. The procedure is based on the compressive sensing $(C S)$ method for the reconstruction of corrupted pixels. It is shown that the proposed procedure can extract watermark with a moderate impulsive noise level. It is well known that most of the images are approximately sparse in the 2D DCT domain. Moreover, we can force sparsity in the watermarking procedure and obtain almost strictly sparse image as a desirable input to the CS based reconstruction algorithms. Compared to the state-of-the-art methods for impulse noise removal, the proposed solution provides much better performance in watermark extraction.
\end{abstract}

\section{Keywords}

Image watermarking, impulsive noise, compressive sensing, sparse reconstruction, gradient algorithm

\section{Introduction}

Digital watermarking techniques are introduced to protect digital multimedia content mostly in the sense of authorship protection, authentication or integrity protection [1-3]. Generally, watermarking is implemented by inserting a certain sequence (watermark) to the existing digital content.

The sequence is embedded in spatial or some transformation domain like the discrete cosine transform (DCT) [1], [3], the discrete wavelet transform (DWT) [1], [4], time-frequency or space/spatial-frequency transformation [1], [2], [5]. When considering invisible and robust watermarking scenario, it has been shown that the transform domain based approaches overcome the performance of time or spatial domain techniques. Embedding another image (logo image) into the original one is a special case of the watermarking.

If the watermarked image is corrupted by the impulsive noise the watermark detection is deteriorated. For instance, using median filtering as a common method for removing impulse noise will completely degrade (or remove) the watermark. Some recently developed denoising techniques [6], [7] restore high-quality images even with a high noise level.
An efficient two stage adaptive method for restoration of images corrupted by impulse noise is proposed in [6]. Therein, the restoration is based on the mean value of the neighboring uncorrupted pixels, showing improved performance comparing with the other state-of-the-art techniques. Another interesting approch presented in [7] is based on adaptive median filtering and block matching and 3D filtering (BM3D) scheme. All of these methods provide high structural similarity index (SSIM) and good peak signal-to-noise ratio (PSNR), between the non-noisy and reconstructed image, but still the embedded watermark cannot be recovered from the resulting image even when the low noise intensity is applied at the input.

In this paper, we propose a procedure for watermark detection from the corrupted images. The procedure is based on the compressive sensing (CS) [8], [9] reconstruction algorithms. Namely, by exploiting the image sparsity property and the advantages of CS reconstruction approach, it is possible to achieve high watermark detectability beside the high quality of restored image. Unlike the classical restoration techniques that are mainly based on a certain kind of interpolation (filtering) thus dealing with small interpolation errors, the CS-based algorithms are able to restore the exact values of corrupted pixels under some conditions. Here, we employ the recently proposed gradient descent reconstruction algorithm [10], [11], while the sparsity is enforced in the 2D DCT domain.

We will assume that the corrupted pixels positions are known. In the case of impulsive noise, the corrupted pixels can be detected by using statistical tools. Moreover, recent research shows that corrupted pixels can be accurately detected by examining sparsity measure [13].

The presented approach could be extended to other sparsity domains like the discrete Fourier domain or wavelet domain. Generally speaking, any linear transform of an image that satisfies the sparsity property can be applied following the same principles as in the case of the DCT. However, the watermark insertion, image reconstruction, and watermark detection procedures should be adapted to the specific transform domain, since different domains have their own properties. The 2D DCT is considered here as the most com- 
monly used domain in image analysis and definitely one of the widely explored in watermarking applications.

The main contribution of the paper is the improvement of watermark detection in the case of heavy impulse noise. The robustness to other common attacks has not been changed with the proposed detection procedure compared to the standard 2D DCT watermarking. Particularly, the DCT based watermarking is generally robust to JPEG compression, especially if the watermark is inserted in lower bit planes. In the case of rotation attack, the DCT-based approaches cannot retrieve the watermark without additional patterns used for the inverse transform. Finally, instead of filtering (e.g. median filtering procedure), here we propose to use a total reconstruction approach providing the improvement of results.

The paper is structured as follows. In Sec. 2 a brief theoretical introduction to the $\mathrm{CS}$ reconstruction is given. The proposed method is introduced in Sec. 3. The examples and statistical analysis are presented in Sec. 4.

\section{CS Theory and Reconstruction}

The signal is sparse if the number of its non-zero values $K$ in a certain representation domain is much smaller than a total number of signal values $N$. Sparse signal vector will be denoted by $\mathbf{X}$. The signal is measured and $M$ observed measurements are expressed as a linear combination of coefficients in $\mathbf{X}$. Measurements are stored in vector $\mathbf{y}$. Relation between measurements and sparse coefficients is

$$
\mathbf{y}=\mathbf{A X}
$$

where $\mathbf{A}$ is an $M \times N$ measurement matrix.

It is shown that, under some conditions [8], [9], sparse signal $\mathbf{X}$ can be uniquely reconstructed from the available measurements. This is classical CS reconstruction problem that can be formulated as a following minimization problem

$$
\min \|\mathbf{X}\|_{0}, \quad \text { subject to } \mathbf{y}=\mathbf{A X}
$$

where $\|\mathbf{X}\|_{0}$ denotes the number of non-zero coefficients in $\mathbf{X}$, and it is called $\ell_{0}$ pseudo-norm (or $\ell_{0}$ norm) although it does not satisfy basic norm properties.

Minimization procedure can be performed by using a direct search procedure assuming that at most $K$ coefficients in $\mathbf{X}$ are nonzero and analyzing all possible positions of these coefficients. If there is only one combination satisfying the constraint, then the reconstruction problem is solved and a solution is unique. This approach is not computationally feasible since the total number of combinations $\left(\begin{array}{l}N \\ K\end{array}\right)$ is too large.

Another approach is to formulate minimization problem in a convex form. The $\ell_{1}$ norm is a closest convex approximation of the $\ell_{0}$ pseudo-norm. The problem formulation becomes

$$
\min \|\mathbf{X}\|_{1}, \quad \text { subject to } \mathbf{y}=\mathbf{A X} \text {. }
$$

It is shown that, under some conditions, problem (3) have the same solution as (2) [8], [9]. There are several reconstruction techniques that have been developed for different scenarios [10], [11], [14], [15]. Without loss of generality, we will use recently proposed gradient-based reconstruction method [10], [11]. This method does not change values of the available samples (pixels) and use finite approximation of the sparsity measure gradient for correction of the corrupted samples. The gradient approximation is obtained by varying current value of each corrupted sample by adding and subtracting some finite value $\Delta$. The algorithm performance is fast, provides accurate solution and does not assume the strict sparsity constraint, which is highly important in practical applications.

It is known that most real-word images are sparse (or approximately sparse) in the 2D DCT domain [2], [13]. The image pixels can be considered as measurements of the 2D DCT of the image. In this case complete (full) set of measurements is well defined (all image pixels form a full set of measurements). If some pixels are missing or corrupted, we are dealing with the reduced set of measurements and CS reconstruction techniques could be applied in order to reconstruct missing (corrupted) pixels.

Since the image, and the corresponding 2D DCT, are two-dimensional signals they should be rearranged in a vector form in order to reduce pixel reconstruction to formulation (3). The measurement vector $\mathbf{X}$ is obtained by stacking 2D DCT columns, and measurement matrix $\mathbf{A}$ is obtained from the corresponding 2D DCT weighting coefficients. The measurements vector $\mathbf{y}$ contains values of all available (uncorrupted) image pixels.

\section{Proposed Watermarking Method}

\subsection{Watermarking Procedure}

The original image is divided into $B \times B$ blocks, where $B$ is block size. The 2D DCT is calculated for each block. A set of coefficients suitable for watermark embedding is selected. The watermark sequence is then embedded into a given bit-plane of the selected 2D DCT coefficients.

For coefficients selection we define a binary matrix $\mathbf{R}$ of order $B \times B$, with the coefficients given by

$$
R_{i, j}= \begin{cases}1, & \text { for } d_{1} \leq i+j-1 \leq d_{2} \\ 0, & \text { otherwise }\end{cases}
$$

for $i, j=1,2, \ldots, B$, where $d_{1}$ and $d_{2}$ are starting and ending anti-diagonal filled with ones in matrix $\mathbf{R}$. The coefficient selection matrix aims to avoid low and high frequency parts of the 2D DCT block. Therefore the number of selected coefficients $N_{\text {sel }}$ is

$$
N_{\text {sel }}=\sum_{k=d_{1}}^{d_{2}} \min \{k, 2 B-k\}
$$


where the number of elements on the $k$-th anti-diagonal of matrix $\mathbf{R}$ is $\min \{k, 2 B-k\}$ for $k=1,2, \ldots, 2 B-1$. As an illustration, for the block size $4 \times 4, d_{1}=3$ and $d_{2}=4$ the selection matrix is

$$
\mathbf{R}=\left[\begin{array}{llll}
0 & 0 & 1 & 1 \\
0 & 1 & 1 & 0 \\
1 & 1 & 0 & 0 \\
1 & 0 & 0 & 0
\end{array}\right]
$$

with 7 selected coefficients out of 16 coefficients in total.

The coefficients selection procedure is repeated for each block resulting in a set of the coefficients that will be used for watermarking.

The watermark is inserted in the bit-plane $b$ of the selected coefficients by replacing $b$-th bit of each coefficient with corresponding watermark bit [2, p. 205-210]. Namely, the watermark is a binary sequence and could also represent a logo image. The total length of watermark should not exceed a total number of the selected coefficients in all blocks. If the total number of selected coefficients is higher than the watermark length, the watermark is repeated to cover all selected coefficients.

The strict sparsity property is preferable for the exact CS reconstruction of corrupted pixels. Prior to the watermark embedding, we can sparsify 2D DCT of each block by setting coefficients below some predefined threshold $T$ to 0 . This is a small but important modification of the classical watermark insertion scheme [2]. The threshold $T$ should be set in a way to avoid significant image degradation. Also, the sparsity can be enhanced by applying image compression to neglect certain 2D DCT values, as in the case of JPEG compression algorithm.

Watermarked image is obtained by applying inverse 2D DCT on each block. The watermark insertion procedure is described in Algorithm 1.

\subsection{Watermark Detection}

It is possible that some pixels of the watermarked image are corrupted. The corrupted pixels can significantly degrade watermark detection, especially when the watermarking is performed in the transformation domain. Two common noise types are Gaussian and impulse noise. Impulse noise can appear in the watermarked image as a consequence of channel transmission errors via noisy communication channels or due to memory cell faults in digital image storage [2, p. 174175], [12, p. 435-439]. One of the frequently analyzed cases of impulse noise is salt $\&$ pepper noise. In this case, some randomly positioned pixels are changed to black (value 0) or white (value 255 for 8-bit gray-scale image). The corrupted pixels can be easily detected in this case. Recent research [13] propose CS based technique for corrupted pixels detection within a full range of pixel values (from 0 to 255 for 8-bit images).

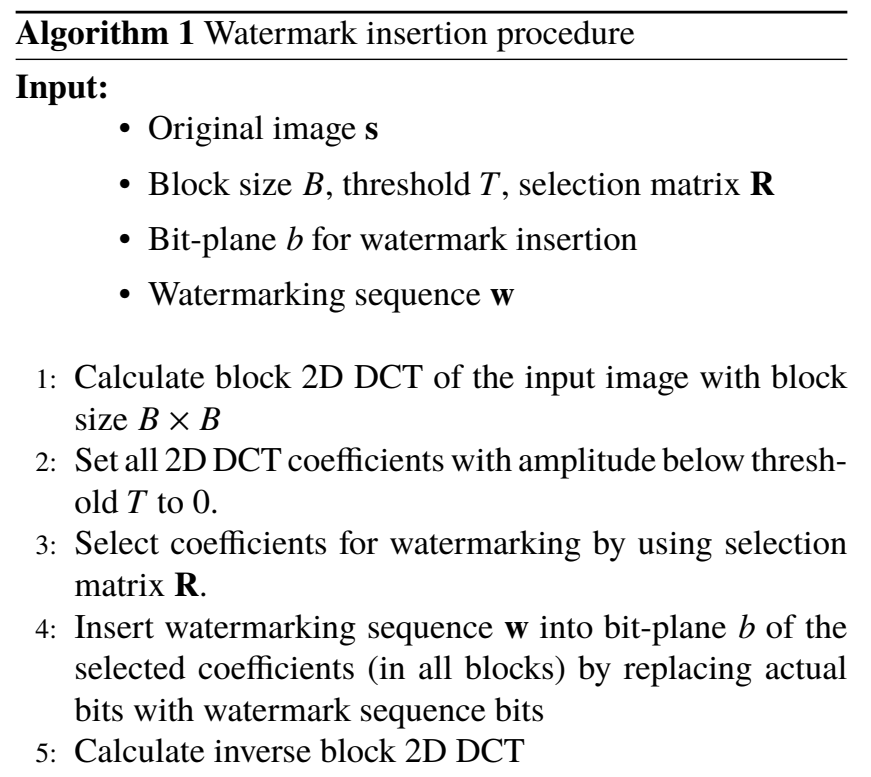

\section{Output:}

- Watermarked image $\mathbf{s}_{\mathrm{W}}$

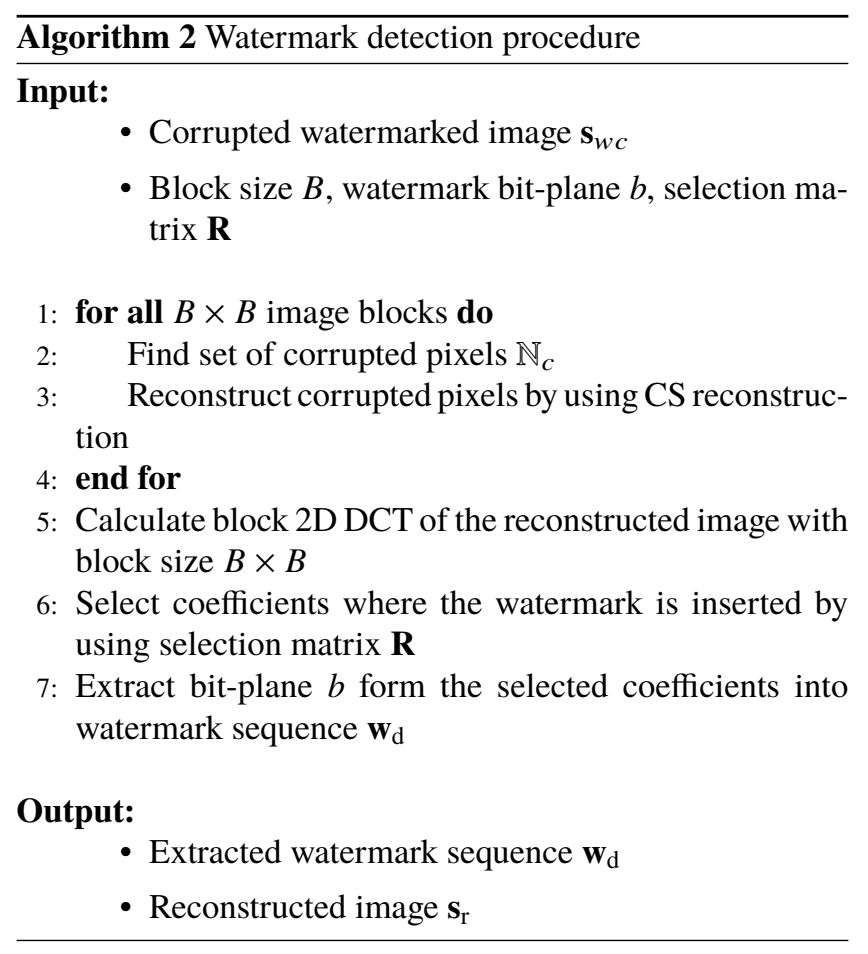

If the watermarked image is corrupted with impulsive noise, image restoration should be applied prior to watermark extraction. Here we will use the CS based procedure for reconstruction of the missing pixels proposed in [10], [11]. The reconstruction code is available at http://www.tfsa.ac.me/pap/sup-001179.zip.

In the first stage, for each $B \times B$ block, the set of corrupted pixels within selected block is estimated. Then, the $\mathrm{CS}$ reconstruction procedure is applied. 
After image restoration, the watermark sequence is extracted as $b$-th bit-plane of the selected 2D DCT coefficients [2]. If the number of selected coefficients is higher than watermark length, several copies of watermark sequence are obtained.

The image restoration and watermark detection procedure are described in Algorithm 2.

\subsection{Performance Measures}

Since image reconstruction is performed prior to watermark detection we will use peak signal-to-noise ratio (PSNR) and structural similarity index (SSIM) as performance measures. For $N \times M$ images $\mathbf{s}_{\mathrm{w}}$ and $\mathbf{s}_{\mathrm{r}}$ the PSNR is defined as

$$
\text { PSNR }=10 \log _{10} \frac{255^{2}}{\frac{1}{N M} \sum_{i=1}^{M} \sum_{j=1}^{N}\left[s_{w}(i, j)-s_{r}(i, j)\right]^{2}}
$$

The SSIM is proposed in [16] as universal image quality index used to measure the similarity between two images. It is based on means, variances, and covariance of the corresponding images. The SSIM take values from 0 to 1 , where 1 means that there is no difference between compared images.

Watermark detection quality is measured using the error-bit-rate (EBR). It is the ratio between the number of wrongly detected bits and the total number of bits in the watermark sequence. For binary watermark sequence $\mathbf{w}$ of length $N_{\mathrm{w}}$ and detected watermark sequence $\mathbf{w}_{\mathrm{d}}$ it is calculated (in percents) as

$$
\mathrm{EBR}=100 \frac{\sum_{i=1}^{N_{\mathrm{w}}}\left|w(i)-w_{\mathrm{d}}(i)\right|}{N_{\mathrm{w}}}
$$

\section{Examples}

The proposed procedure is applied to $512 \times 5128$-bit gray-scale image "Lena". The chosen watermarking image is 1-bit $26 \times 10$ image "LOGO". The proposed watermarking procedure is applied with block size $B=32$, threshold $T=40$ and third bit-plane $(b=3)$ is used for watermark embedding. The original, watermarked, and logo image are presented in Fig. 1. The PSNR in the watermarked image $\mathbf{S}_{\mathrm{W}}$ is $31.1 \mathrm{~dB}$ (with original image $\mathbf{s}$ as reference) and SSIM is 0.815 .

The watermarked image is corrupted with salt \& pepper noise. Noise intensity is $6 \%$ for upper subplots in Fig. 2 and $20 \%$ for lower subplots in Fig. 2. Corrupted images are presented in the first column of Fig. 2. The detected watermark image is presented in all cases. We can see that watermark detection from the corrupted images is not possible.

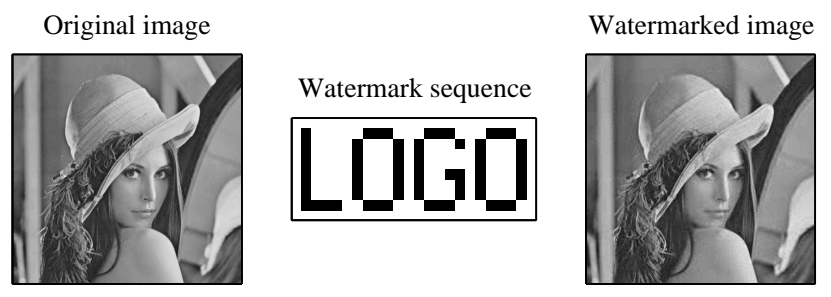

Fig. 1. Watermark detection example. Original image $(512 \times$ $512)$, enlarged logo image $(26 \times 10)$ and watermarked image are presented.
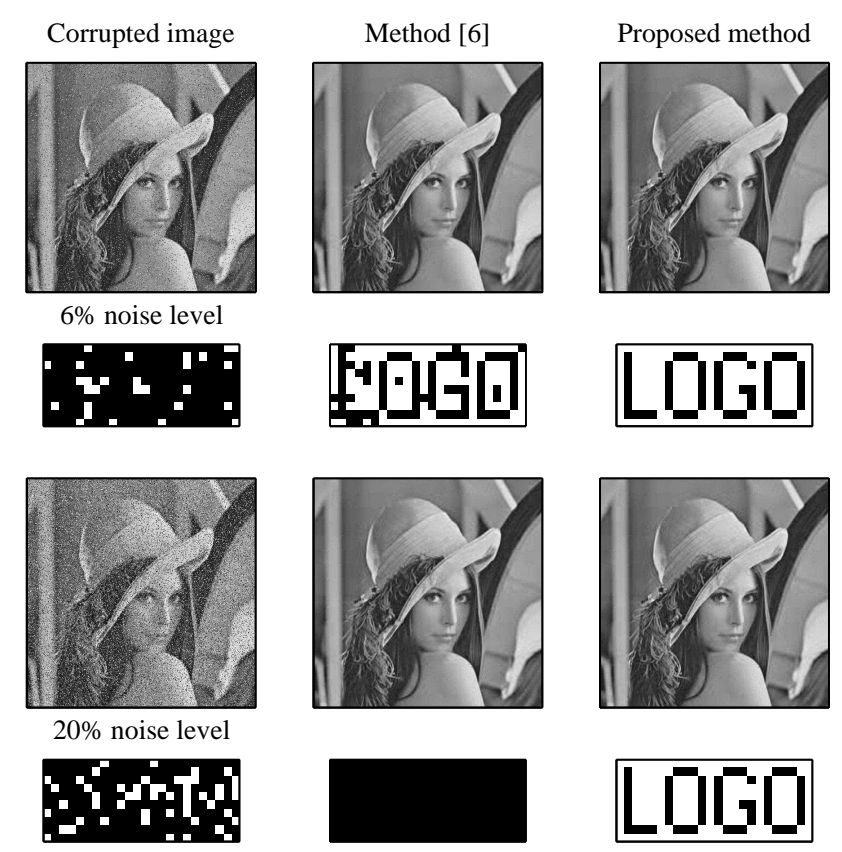

Fig. 2. Watermark detection from the image corrupted by salt $\&$ pepper noise with $6 \%$ and $20 \%$ of corrupted pixels. The detection results for noisy image are presented in the first column, the results with restoration proposed in [6] are in the second column and the results obtained by the proposed method are in the third column. The detected LOGO sequences are given below images.

Next, we apply restoration method presented in [6]. For $6 \%$ noise level, the PSNR in the reconstructed image $\mathbf{s}_{\mathrm{r}}$ is $48.4 \mathrm{~dB}$ (with uncorrupted watermarked image $\mathbf{s}_{\mathrm{w}}$ as reference). Corresponding SSIM is 0.9976. For $20 \%$ noise level, the PSNR of $42.5 \mathrm{~dB}$ and SSIM 0.9914 are obtained. This method achieves high PSNR and SSIM, so the reconstructed images (second column in Fig. 2) quality is good. However, when we try do detect the watermark, for $6 \%$ corrupted pixels we obtain "LOGO" image with some wrong pixels. In the case of $20 \%$ corruption, the watermark detection is not possible.

The method proposed in this paper include CS based reconstruction of the corrupted pixels. The reconstructed images are presented in Fig. 2, the third column. The achieved PSNR is $65.0 \mathrm{~dB}$ ans SSIM 0.9998 for $6 \%$ corrupted pixels. For $20 \%$ corrupted pixels the PSNR is $59.3 \mathrm{~dB}$ and SSIM 


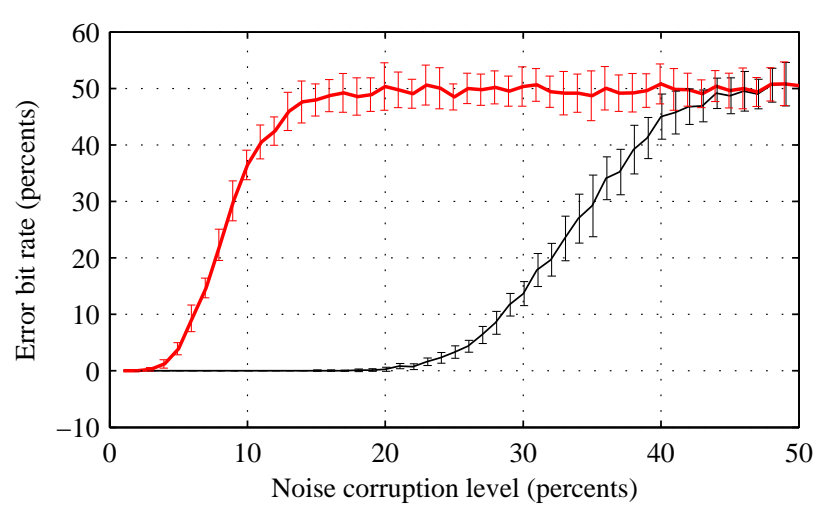

Fig. 3. Error bit rate for varying noise corruption level. Proposed method - thin black line, method [6] - thick red line. The results are obtained by averaging over 20 realizations. Standard deviation is calculated and presented with vertical bars.

\begin{tabular}{c|ccc|ccc} 
Noise & \multicolumn{3}{|c|}{ Proposed method } & \multicolumn{3}{c}{ Method in [6] } \\
level & EBR & PSNR & SSIM & EBR & PSNR & SSIM \\
\hline $1 \%$ & 0.00 & 73.03 & 1.0000 & 0.00 & 56.41 & 0.9996 \\
$2 \%$ & 0.00 & 70.05 & 0.9999 & 0.00 & 53.28 & 0.9993 \\
$3 \%$ & 0.00 & 68.32 & 0.9999 & 0.27 & 51.50 & 0.9989 \\
$4 \%$ & 0.00 & 66.95 & 0.9999 & 1.23 & 50.20 & 0.9985 \\
$5 \%$ & 0.00 & 66.01 & 0.9999 & 3.92 & 49.22 & 0.9981 \\
$6 \%$ & 0.00 & 65.22 & 0.9998 & 9.31 & 48.32 & 0.9978 \\
$7 \%$ & 0.00 & 64.56 & 0.9998 & 14.69 & 47.65 & 0.9974 \\
$8 \%$ & 0.00 & 63.94 & 0.9998 & 22.31 & 47.04 & 0.9970 \\
$9 \%$ & 0.00 & 63.39 & 0.9998 & 30.10 & 46.46 & 0.9966 \\
$10 \%$ & 0.00 & 62.93 & 0.9997 & 36.46 & 45.92 & 0.9961 \\
$15 \%$ & 0.04 & 61.10 & 0.9996 & 47.96 & 44.03 & 0.9941 \\
$20 \%$ & 0.29 & 59.69 & 0.9995 & 50.35 & 42.55 & 0.9917 \\
$25 \%$ & 3.31 & 58.60 & 0.9993 & 48.48 & 41.29 & 0.9890 \\
$30 \%$ & 13.67 & 57.57 & 0.9991 & 50.33 & 39.89 & 0.9853 \\
$35 \%$ & 29.21 & 56.57 & 0.9989 & 48.71 & 38.39 & 0.9802 \\
$40 \%$ & 45.02 & 55.56 & 0.9987 & 50.79 & 36.25 & 0.9695 \\
$45 \%$ & 48.69 & 54.64 & 0.9984 & 49.63 & 34.09 & 0.9510 \\
$50 \%$ & 50.48 & 53.17 & 0.9979 & 50.48 & 33.76 & 0.9434 \\
\hline
\end{tabular}

Tab. 1. Watermark detection and image reconstruction results. Error bit rate in the detected watermark, PSNR, and SSIM for reconstructed images is given for various noise levels.

is 0.9994 . That is significantly better than reconstruction method [6]. We can see that in both cases we detect the watermark with no errors.

In the next experiment, we vary noise intensity from $1 \%$ to $50 \%$. Image "Lena" is used here and the watermarking sequence was randomly generated $26 \times 101$-bit image. The PSNR and SSIM are used as a measure of the reconstruction quality. The EBR in the detected watermark is used as a measure of detection quality. The results are presented in Tab. 1 and Fig. 3. The mean value of EBR, PSNR, and SSIM over 20 random realizations of watermark sequence and noise are presented in Tab. 1. The mean value and standard deviation of the EBR are plotted in Fig. 3. We can see that the proposed technique achieves better EBR, PSNR, and SSIM compared to [6]. The watermark can be successfully detected for noise level up to $25 \%$ while, the restoration method proposed in [6] fails to detect watermark for noise level above $9 \%$.

\begin{tabular}{c|ccc|ccc} 
Test & \multicolumn{3}{|c|}{ Proposed method } & \multicolumn{3}{c}{ Method in $[6]$} \\
image & EBR & PSNR & SSIM & EBR & PSNR & SSIM \\
\hline$\# 1$ & 0.00 & 59.29 & 0.9996 & 46.25 & 31.95 & 0.9682 \\
$\# 2$ & 0.00 & 59.48 & 0.9995 & 52.50 & 41.13 & 0.9864 \\
$\# 3$ & 0.00 & 56.98 & 0.9994 & 47.08 & 36.58 & 0.9755 \\
$\# 4$ & 0.00 & 59.29 & 0.9995 & 54.17 & 42.75 & 0.9882 \\
$\# 5$ & 0.00 & 59.48 & 0.9995 & 42.50 & 44.66 & 0.9924 \\
$\# 6$ & 0.00 & 56.61 & 0.9994 & 50.00 & 36.10 & 0.9739 \\
$\# 7$ & 0.00 & 48.51 & 0.9994 & 50.00 & 28.10 & 0.9585 \\
$\# 8$ & 1.67 & 60.03 & 0.9995 & 47.92 & 42.61 & 0.9905 \\
$\# 9$ & 0.00 & 55.94 & 0.9994 & 60.00 & 33.45 & 0.9757 \\
$\# 10$ & 0.83 & 59.87 & 0.9995 & 47.08 & 38.65 & 0.9898 \\
$\# 11$ & 0.00 & 45.15 & 0.9985 & 52.08 & 25.94 & 0.9299 \\
$\# 12$ & 0.00 & 54.74 & 0.9992 & 55.00 & 35.16 & 0.9674 \\
$\# 13$ & 0.00 & 59.10 & 0.9996 & 45.83 & 36.49 & 0.9846 \\
$\# 14$ & 0.00 & 52.68 & 0.9997 & 45.42 & 30.12 & 0.9539 \\
$\# 15$ & 0.00 & 56.55 & 0.9996 & 52.92 & 34.15 & 0.9717 \\
$\# 16$ & 0.00 & 54.27 & 0.9995 & 50.42 & 32.62 & 0.9579 \\
$\# 17$ & 0.00 & 55.17 & 0.9994 & 45.00 & 31.10 & 0.9533 \\
$\# 18$ & 0.00 & 45.99 & 0.9989 & 44.17 & 28.09 & 0.9417 \\
$\# 19$ & 0.00 & 56.81 & 0.9994 & 42.92 & 37.02 & 0.9783 \\
$\# 20$ & 0.00 & 58.85 & 0.9996 & 42.50 & 37.37 & 0.9795 \\
$\# 21$ & 2.92 & 60.00 & 0.9995 & 50.83 & 39.38 & 0.9842 \\
$\# 22$ & 0.83 & 53.84 & 0.9995 & 49.17 & 33.55 & 0.9742 \\
$\# 23$ & 5.00 & 60.25 & 0.9994 & 50.42 & 44.46 & 0.9925 \\
$\# 24$ & 2.50 & 60.03 & 0.9995 & 43.75 & 42.24 & 0.9890 \\
$\# 25$ & 9.17 & 59.42 & 0.9993 & 48.33 & 43.59 & 0.9878 \\
\hline & & & & & &
\end{tabular}

Tab. 2. Results obtained for $20 \%$ corrupted pixels for standard gray-scale test images database.

The proposed procedure is statistically tested on 49 standard test images (available online at http://descai.ugr.es/cvg/CG/base.htm). The results obtained with test images 1-25 are given in Tab. 2. Noise intensity was $20 \%$. We can see that the proposed technique provide EBR below $10 \%$ in all presented cases, while for most images the EBR is 0 .

\section{Conclusion}

Watermark detection form the images corrupted by impulsive noise is analyzed. It is shown that state-of-the-art reconstruction methods could not be applied when the noise level is high. Although the reconstructed images visual quality is high, the watermark is significantly degraded by filtering. The proposed technique is based on the CS reconstruction of the corrupted pixels. It is demonstrated on examples and tested on standard test images database. It is shown that the proposed technique is able to extract watermark even from heavily corrupted images (with up to $25 \%$ of corrupted pixels).

\section{Acknowledgments}

This work is supported by the Montenegrin Ministry of Science, project grant funded by the World Bank loan: CSICT "New ICT Compressive sensing based trends applied to: multimedia, biomedicine and communications". 


\section{References}

[1] BARNI, M., BARTOLINI, F. Watermarking Systems Engineering. New York, NY (USA): Marcel Dekker, Inc., 2004. ISBN: 978-0824748067

[2] STANKOVIĆ, S., OROVIĆ, I., SEJDIĆ, E. Multimedia Signals and Systems: Basic and Advance Algorithms for Signal Processing. 1st ed. New York, NY (USA): Springer-Verlag, 2015. ISBN: 978-3-319-23948-4

[3] FURHT, B., KIROVSKI, D.. Multimedia Watermarking Techniques and Applications. Boca Raton, FL (USA): Auerbach Publication, 2006. (Chapter 3: MUHAREMAGIĆ, E., FUHRT, B. Survey of Watermarking Techniques and Applications) ISBN: 9780849372131

[4] LIU, J., SHE, K., WU, H. Blind image watermarking using dual embedding scheme in the wavelet transform domain. Journal of Computational Information Systems, 2010, vol. 6, no. 6, p. 1887-1896. ISSN: 1553-9105

[5] STANKOVIĆ, S., OROVIĆ, I., CHABERT, M., et al. Image watermarking based on the space/spatial-frequency analysis and Hermite functions expansion. Journal of Electronic Imaging, 2013, vol. 22, no. 1. DOI: 10.1117/1.JEI.22.1.013014

[6] RAMADAN, Z. M. Efficient restoration method for images corrupted with impulse noise. Circuits, Systems, and Signal Processing, 2012, vol. 31, no. 4, p. 1397--1406. DOI: 10.1007/s00034-011-9380-z

[7] DJUROVIĆ, I. BM3D filter in salt-and-pepper noise removal. EURASIP Journal on Image and Video Processing, 2016, vol. 2016, no. 13, p. 1-11. DOI: 10.1186/s13640-016-0113-x

[8] CANDÈS, E. J., ROMBERG, J., TAO, T. Robust uncertainty principles: Exact signal reconstruction from highly incomplete frequency information. IEEE Transactions on Information Theory, 2006, vol. 52, no. 2, p. 489-509. DOI: 10.1109/TIT.2005.862083

[9] BARANIUK, R. Compressive sensing. IEEE Signal Processing Magazine, 2007, vol. 24, no. 4, p. 118-121. DOI: 10.1109/MSP.2007.4286571

[10] STANKOVIĆ, L., DAKOVIĆ, M., VUJOVIĆ, S. Adaptive variable step algorithm for missing samples recovery in sparse signals. IET Signal Processing, 2014, vol. 8, no. 3, p. 246-256. DOI: 10.1049/iet-spr.2013.0385

[11] STANKOVIĆ, L., DAKOVIĆ, M. On a gradient-based algorithm for sparse signal reconstruction in the signal/measurements domain. Mathematical Problems in Engineering, 2016, vol. 2016, p. 1-11. DOI: $10.1155 / 2016 / 6212674$

[12] YAROSLAVSKY, L. P. Theoretical Foundations of Digital Imaging Using MATLAB. CRC Press, 2012. ISBN: 978-1439861400

[13] STANKOVIĆ, I., OROVIĆ, I., STANKOVIĆ, S., et al. Iterative denoising of sparse images. In Proceedings of the 39th International Convention on Information and Communication Technology, Electronics and Microelectronics (MIPRO). May 2016, p. 503-507. DOI: 10.1109/MIPRO.2016.7522196

[14] TROPP, J. A., GILBERT, A. C. Signal recovery from random measurements via orthogonal matching pursuit. IEEE Transactions on Information Theory, 2007, vol. 53, no. 12, p. 4655-4666. DOI: 10.1109/TIT.2007.909108

[15] NEEDELL, D., TROPP, J. A. CoSaMP: Iterative signal recovery from incomplete and inaccurate samples. Communications of the ACM, 2010, vol. 53, no. 12, p. 93-100. DOI: 10.1145/1859204.1859229

[16] WANG, Z., BOVIK, A. C., SHEIKH, H. R., et al. Image quality assessment: From error visibility to structural similarity. IEEE Transactions on Image Processing, 2004, vol. 13, no. 4, p. 600-612. DOI: 10.1109/TIP.2003.819861

\section{About the Authors...}

Budimir LUTOVAC was born in Berane, Montenegro. He received the B.Sc. and M.Sc. degrees in electrical engineering from the University of Montenegro in 1992 and 1996 respectively. He received his Ph.D. in Electrical Engineering in 2003 at the University of Belgrade, Serbia. He is currently an Associate professor at the University of Montenegro. His research interests include symbolic analysis, synthesis of electrical (microwave) circuits and digital filters.

Miloš DAKOVIĆ was born in 1970 in Nikšić, Montenegro. He received a B.S. in 1996, an M.S. in 2001, and a Ph.D. in 2005, all in electrical engineering from the University of Montenegro. He is an associate professor at the University of Montenegro. His research interests are in signal processing, time-frequency signal analysis, compressive sensing, and radar signal processing. He is a member of the Time-Frequency Signal Analysis Group (www.tfsa.ac.me) at the University of Montenegro, where he is involved in several research projects.

Srdjan STANKOVIĆ (M'94-SM'08) received M.S. degree from the University of Zagreb, Croatia, in 1991, and the Ph.D. degree from the University of Montenegro (UoM) in 1993, both in Electrical Engineering (EE). In 1992 he joined the Faculty of EE, UoM, where he is currently a Full Professor. In the period 2007-2013 he served as Dean of this Faculty. His interests are in signal processing and multimedia systems. He is a member of the Board of Directors in Montenegrin Broadcasting Company since 2004, where he was also the President (2005- 2006). In 1998 he as at the Department of Informatics, Aristotle University, Thessaloniki. In the 1999-2000, he was on leave at the Darmstadt University of Technology, with the Signal Theory Group, and in 2002 he spent three months at the Department of Computer Science, University of Applied Sciences Bonn-Rhein-Sieg, supported by the AvH Foundation. From 2004 to 2010, he had research stays with the E3I2 Lab, ENSIETA, Brest, France, Center for DSP research at King's College London, Laboratory of Mathematical Methods in Image Processing, at Moscow State Lomonosov University, GIPSA Laboratory at INPG Grenoble. He spent academic 2012/2013 with the Center for Advanced Communications at the Villanova University, PA. He published a book "Multimedia Signals and Systems" by Springer and several textbooks on electronics devices (in Montenegrin). He published more than 200 journal and conference papers. In 2010, he was the Lead Guest Editor of the EURASIP Journal on Advances in Signal Processing for the special issue: Time-frequency analysis and its applications to multimedia signals, as well as a Guest Editor of the Signal Processing for special issue: Fourier related transforms. He is the Lead Guest Editor of the IET Signal Processing for the Special issue: Compressive Sensing and Robust Transforms. From 2005 to 2009 he was serving 
as an Associate Editor of the IEEE Transactions on Image Processing.

Irena OROVIĆ was born in Montenegro, in 1983. She received the B.Sc., M.Sc., and Ph.D. degrees in electrical engineering from the University of Montenegro (UoM), in 2005, 2006, and 2010, respectively. From 2005 to 2010, she was a TA with the UoM. Since 2010, she has been an Assistant Professor with the Faculty of EE, UoM. She finished her Diploma project in ENSIETA Brest, France. She received the Award of the city Podgorica as the best student at the Faculty of EE, UoM in 2003, the Award of Montenegrin Academy of Science and Arts (CANU) in 2004, the Award for the best student of natural and technical sciences at the UoM 2005, TRIMO Awards Slovenia - Award for the best PhD thesis in
2010, Award for the Best Woman Scientist in Montenegro, Ministry of Science of Montenegro, 2011. She has spent a period of time in ENSIETA Bresta, France during 2005 and 2006. In 2007 she spent one month at the University Bonn-Rhien Sieg in Bonn, Germany. During 2008 and 2009 she stayed several times at INPG Grenoble, France (2008 and 2009), and during 2010 and 2011 within the Villanova Univerzitet USA. Dr Orovic has published more than 60 papers in the leading scientific journals and conferences. As a coauthor she has published 6 books. She is Vice President of the Council for Scientific Research Activity on Montenegro. Her research interests include compressive sensing, multimedia signals and systems, and time-frequency analysis with applications. 